\title{
Photoluminescence Studies on Nanocrystalline Tin Oxide Powder for Optoelectronic Devices
}

\author{
L. C. Nehru ${ }^{1}$, V. Swaminathan ${ }^{2}$, C. Sanjeeviraja ${ }^{1, *}$ \\ ${ }^{1}$ School of Physics, Alagappa University, Karaikudi, 630002, India \\ ${ }^{2}$ School of Materials Science and Engineering, Nanyang Technological University
}

\begin{abstract}
Nanocrystalline tin oxide $\left(\mathrm{SnO}_{2}\right)$ powders have been synthesized by a low temperature chemical precipitation method. As-prepared and heated powders were characterized by XRD, SEM and luminescence studies. Crystallographic parameters such as crystallite size, lattice parameters and dislocation density in $\mathrm{SnO}_{2}$ nanocrystalline powders were calculated by Rietveld analysis. The average crystallite size of $9-43 \mathrm{~nm}$ was obtained for $\mathrm{SnO}_{2}$ powders through controlled heat treatment process. The washed powders morphology was almost spherical in shape and average agglomerate crystal size was between $0.2-0.4 \mu \mathrm{m}$. A Photoluminescence (PL) study was measured at an excitation wavelength of $265 \mathrm{~nm}$ for as-prepared and annealed powders; it showed a broad emission peak at $417 \mathrm{~nm}$ for all powders. The highest PL emission was attained for the powder annealed at $500^{\circ} \mathrm{C}$. The synthesized nanocrystalline $\mathrm{SnO}_{2}$ oxide semiconductor material could be suitable for making optoelectronic and sensor devices.
\end{abstract}

Keywords Nano, $\mathrm{SnO}_{2}$, Oxide, Chemical precipitation, Photoluminescence

\section{Introduction}

Over recent years, nanoscience and nanotechnology are attracting and focusing more attention in many commercial applications. In semiconductor nanoparticles, particle size, shape and surface states are the predominant factors, which influences its properties such as optical, magnetic, electric and catalytic[1]. The optical properties with size effect of nanocrystalline semiconductors have been a subject of great interest in recent years. In quantum size effect, when the particle size of a semiconductor is compared to the Bohr radius[2], the ratio of surface atoms to those with the interior enhances the surface properties of the material. Due to these effects, band gap of the semiconductor nanoparticles has been modified, which makes a lot of changes in their electronic properties when compared to the bulk. Thus, there is an increase in the band gap i.e. the visible spectra show a blue shift, which is ascribed to quantum size effect[3]. This stimulated a great interest in both basic and applied research in semiconductor oxide nanomaterials. Recently tremendous R\&D efforts in materials with reduced dimensions, in nanometer scale, have been developed for the fabrication of new miniaturized electronic devices and sensors, which would work more efficiently and selectively as compared to the existing devices so far. Among the

* Corresponding author:

sanjeeviraja@rediffmail.com (C. Sanjeeviraja)

Published online at http://journal.sapub.org/materials

Copyright (C) 2012 Scientific \& Academic Publishing. All Rights Reserved various semiconductor materials, conducting n-type semiconductor tin oxide $\left(\mathrm{SnO}_{2}\right)$ is having a wide band gap of $\mathrm{Eg}$ $=3.6-3.8 \mathrm{eV} \mathrm{[4]} \mathrm{at} \mathrm{room} \mathrm{temperature.} \mathrm{This} \mathrm{conducting} \mathrm{ox-}$ ide is a well known functional material, which can be operative at low temperature with high sensitivity for applications such as gas sensors, transparent conducting electrode, transistor, solar cells, special coating for energy-conversion, low-emissivity windows, and nanoelectronic devices [5-7]. Thus, a great deal of research work has been devoted to the method of synthesizing $\mathrm{SnO}_{2}$ particles of nano sizes in a controllable manner.

Many efforts have been applied to synthesize the $\mathrm{SnO}_{2}$ nanoparticles and nanowhiskers[8] and several methods have been developed for the synthesis of $\mathrm{SnO}_{2}$ nanoparticles like sol-gel route, chemical vapor deposition, thermal decomposition[9], co-precipitation[10], microwave-assisted solution[11], gas phase condensation[12] and laser ablation[13] etc., Preparation of nano-particles of $\mathrm{SnO}_{2}$ at low cost in industrial scale is a challenge in material production. So, using the cheap materials, simple synthesize route and suitable conditions of synthesis are the main requirement for this process. Therefore, the study of influence of various process parameters such as initial solution combination, time and temperature of heat treatments is very important for nanopowder production. The main purpose of the present research is to study the post-annealing temperature effect on nanoparticles size of $\mathrm{SnO}_{2}$ powders synthesized by the low temperature chemical precipitation method. A few publications described the production of $\mathrm{SnO}_{2}$ nanopowders by chemical synthesis. However, it is a challenge to find an 
efficient and simpler way, using low temperature, cost effective, homogeneous and convenient method suitable for large scale production of $\mathrm{SnO}_{2}$ powder with particle size about several nanometers by using this method. However, earlier research of high surface area powders with agglomeration and irregular particle morphology of tin oxide powders were reported. In the present context, it is challenging to overcome the above drawbacks through a controlled chemical precipitation method. Zhijie Li[7] recently reported the room temperature photoluminescence of blue emission with weak intensity. Few reports have been published on the luminescent characteristic of $\mathrm{SnO}_{2}$ nanocrystalline powder by chemical methods. Particle size plays a significant role in optical properties and its emission shift occurs due to the recombination of the electrons with holes in the valence band. Hence, it is another challenge to produce nanomaterials with stronger luminescence property with peak shift. In order to overcome the above drawbacks, we produced $\mathrm{SnO}_{2}$ nanocrystalline powders with desire size by chemical precipitation method which could enhance the photoluminescence property of the material. In the present paper, a systematic study on nanocrystalline $\mathrm{SnO}_{2}$ powder of various sizes by a simple low temperature chemical synthesize route, which can be subsequently processed by different annealing temperature has been presented in detail. Characterization of crystallographic parameters and photoluminescence shift for different annealing temperatures were performed and the results are discussed.

\section{Experimental}

The experimental steps involved in the preparation of $\mathrm{SnO}_{2}$ nanocrystalline powder is shown in Fig.1.

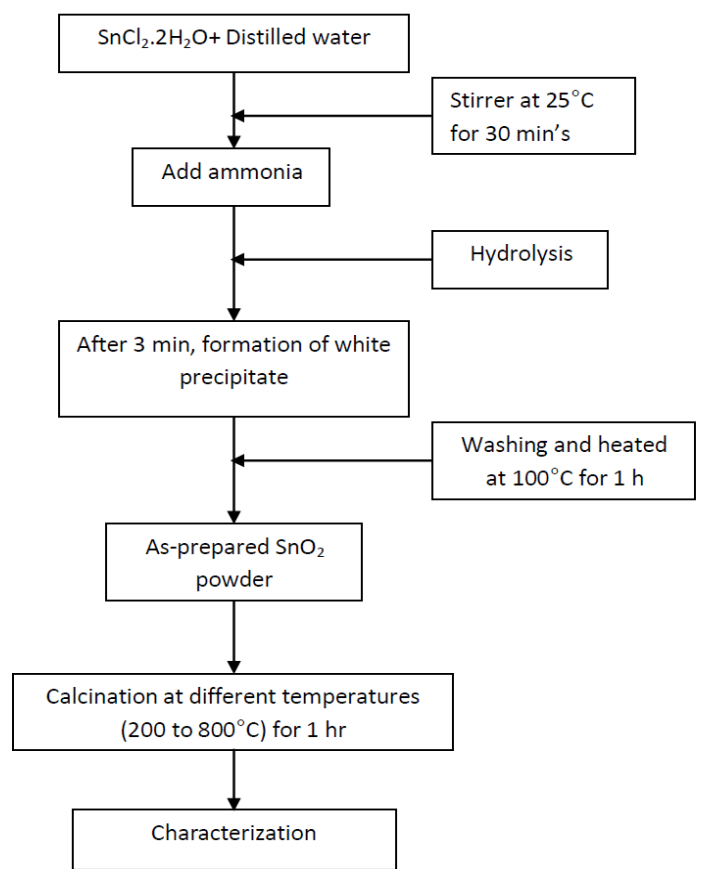

Figure 1. schematic representation of nanocrystalline $\mathrm{sno}_{2}$ powders by chemical precipitate method

\subsection{Characterization}

The as-prepared and annealed nanocrystalline $\mathrm{SnO}_{2}$ powder was characterized by powder X-ray diffraction (XRD) method using a X'Pert PRO PANalytical diffractometer with $\mathrm{CuK} \alpha$ radiation of wavelength $1.5405 \AA$. Diffraction patterns were recorded with a step size of $0.02^{\circ}$, scan speed of $2 \mathrm{~s} / \mathrm{step}$ and scan angle $2 \theta$ from $10^{\circ}$ to $80^{\circ}$. Crystallographic parameters were refined by XRD pattern and Rietveld refinement using TOPAS-3 and Diamond software was used to construct the structural parameters. The dislocation density $(\delta)$, defined as the length of dislocation-lines per unit volume of the crystal can be calculated from $d=1 / \mathrm{D}^{2}$ [14], where $\mathrm{D}$ is the crystallite size and the number of unit cell is calculated from $n=3.14 \times\left({ }^{4} / 3\right) \times\left({ }^{\mathrm{D}} / 2\right)^{3} \times(1 / \mathrm{v})[15]$, where $\mathrm{D}$ is the crystallite size and $\mathrm{V}$ is the cell volume of the $\mathrm{SnO}_{2}$ sample. Morphological of as-prepared and annealed samples was examined by SEM (JEOL-6360). Nanoparticles and diffracted rings pattern was obtained from JEOL 2010 Transmission Electron Microscope. The photoluminescence measurements were carried out from $280 \mathrm{~nm}$ to $480 \mathrm{~nm}$ at room temperature using Varian Cary Eclipse spectrophotometer with $265 \mathrm{~nm}$ as the excitation wavelength of a $15 \mathrm{~W}$ Xenon pulse lamp. All emission spectra were recorded for the detector response and excitation spectra for the lamp profile.

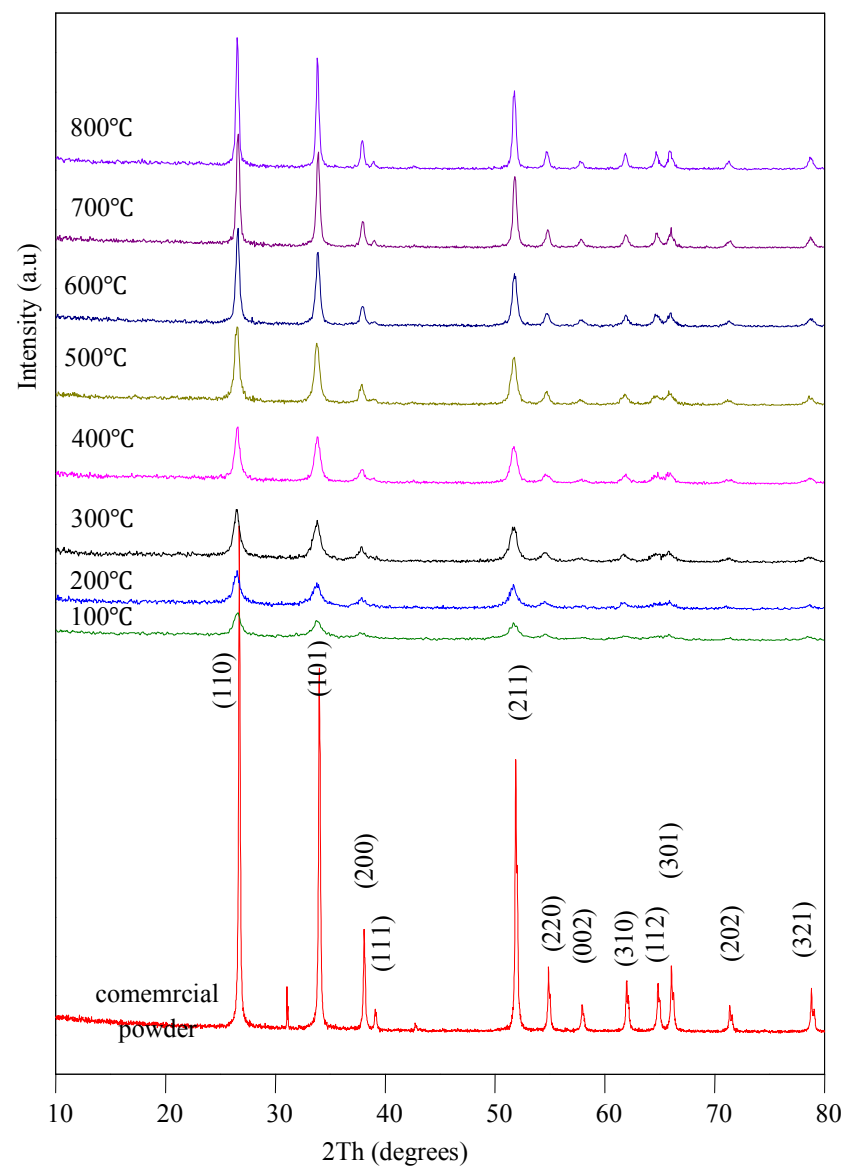

Figure 2. The XRD patterns of $\mathrm{SnO}_{2}$ samples before and after annealed at different temperatures from 200 to $800^{\circ} \mathrm{C}$ and peaks were indexed to the bulk $\mathrm{SnO}_{2}$ (MERCK) with JCPDS no. 88-0287 


\section{Results and Discussion}

Figure 2 shows the XRD patterns of $\mathrm{SnO}_{2}$ nanocrystalline powders for as -prepared and annealed powders at different temperatures from 200 to $800^{\circ} \mathrm{C}$. For as-prepared and annealed powders, the XRD data showed that the tetragonal $\mathrm{SnO}_{2}$ phase was successfully produced, its lattice parameters were $\mathrm{a}=4.7493 \mathrm{~nm}$ and $\mathrm{c}=3.1898 \mathrm{~nm}$ (as-prepared); the lattice constants were comparable with earlier reports.

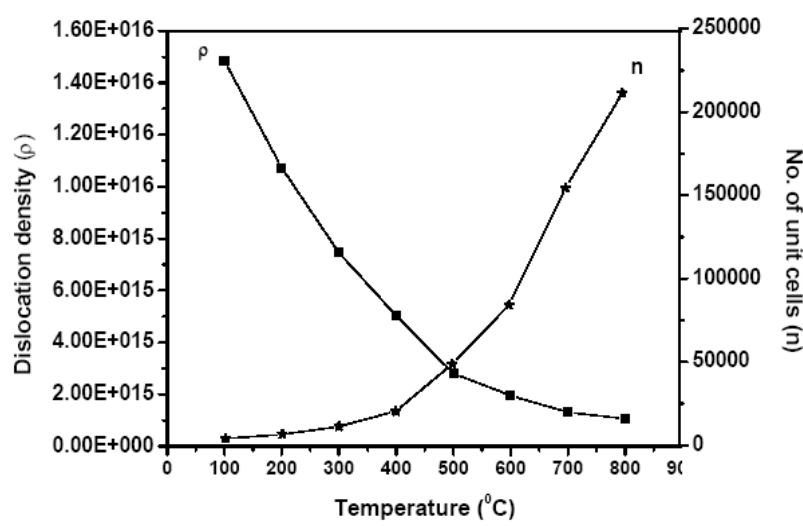

Figure 3. Variation of Dislocation density and number of unit cells as a function of temperature

The obtained pattern showed majority (hkl) peaks of (110), (101), (200), (111), (211), (220), (002), (310), (112), (301), (202) and (321) were corresponding to $\mathrm{SnO}_{2}$ and no traces of unidentified peaks were present in this pattern. From the XRD pattern, as-prepared and annealed nanocrystalline $\mathrm{SnO}_{2}$ powders were compared with bulk commercial (MERCK) powder. The commercial powder pattern showed that the high intense with narrow peaks were observed. The width of the as-prepared Bragg peaks are considerably broadened which indicates a small crystallite size. As the annealing temperature increases from 200 to $800^{\circ} \mathrm{C}$ the intensity of the peaks were increased, where as the FWHM were reduced. The peak broadenings decrease and the intensities of the peaks are gradually sharper with increasing temperatures led to nanocrystalline powders with average crystallite sizes (in $\mathrm{nm}$ ) varying from 8 to $43 \mathrm{~nm}$. The changing of the annealing temperatures from 200 to $800^{\circ} \mathrm{C}$ which decreased the density of nucleation centers as shown in Fig. 3

As the temperature increases, a smaller number of unit cells start to grow, which results in large grain size[16]. A smaller crystallite size exhibits larger cell volume and minimum number of unit cells was present. Thereafter, crystallite size is increased, the cell volume is also decreased due to the heating temperature is increased and the number of unit cells is also increased. The crystallographic parameters were refined using Rietveld analysis of the nanocrystalline $\mathrm{SnO}_{2}$ powders and were shown in Table 1. Crystallite size, cell mass and density, number of unit cells and dislocation density were dependent on thermal conditions. Rietveld method of the $\mathrm{SnO}_{2}$ nanocrystalline powders heat treated at $8000 \mathrm{C}$ was refined (Fig. 4). The weight percentage, $\mathrm{R}$ Bragg and GOF were $\sim 11 \%, \sim 1 \%$ and $\sim 1 \%$ were calculated for as-prepared and heat treated powders (Table1). Using the refined data, the structural model of $\mathrm{SnO}_{2}$ was constructed and its bond length is obtained and comparable with the earlier reports [17-18] (Table 1).

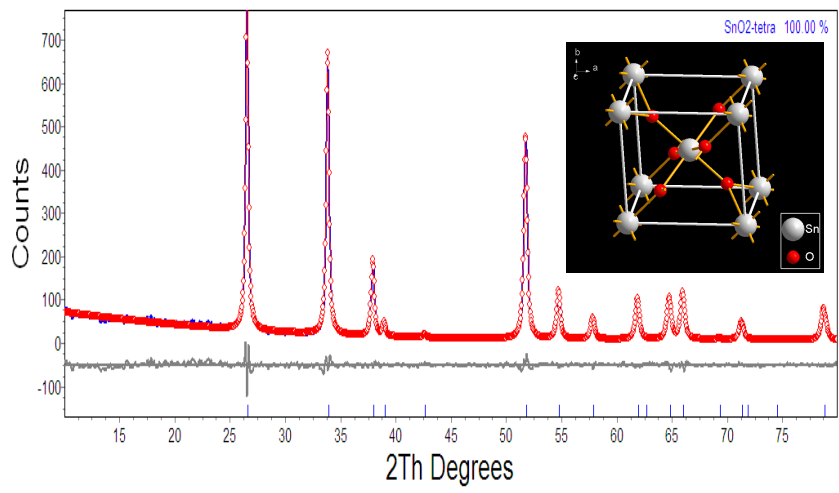

Figure 4. Rietveld refinement of nanocrystalline $\mathrm{SnO}_{2}$ powder annealed at $500^{\circ} \mathrm{C}$ for $1 \mathrm{~h}$

The SEM micrographs (Fig.5), the washed powders morphology are almost spherical in shape and average agglomerate crystal size of $0.2-0.4 \mu \mathrm{m}$. The agglomeration could be due to strong hydrogen bonding in the gel network, which is then difficult to remove in the subsequent stages. The agglomerate size is further increases on heat treatment as shown in Fig. $5 \mathrm{a}, \mathrm{b}$, c and d. The $800^{\circ} \mathrm{C}$ samples, on the contrary, show a better morphology than the $100^{\circ} \mathrm{C}$ samples, have finer particles at the same magnification.
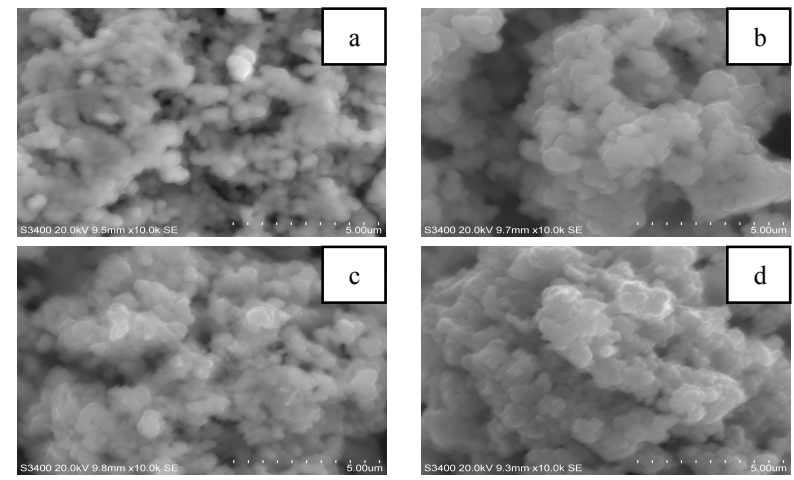

Figure 5. Scanning electron micrographs of nanocrystalline $\mathrm{SnO} 2$ samples at different annealed temperatures (a) as-prepared, (b) $200^{\circ} \mathrm{C}$, (c) $500^{\circ} \mathrm{C}$ and (d) $800^{\circ} \mathrm{C}$

Figure 6 shows a typical excitation and emission spectra of $\mathrm{SnO}_{2}$ nanocrystalline powder prepared by direct chemical precipitation method. The excitation spectrum of the $\mathrm{SnO}_{2}$ shows a strong band at $265 \mathrm{~nm}$ under an emission of $417 \mathrm{~nm}$.

In general, except the sharp excitonic emission, semiconductors have another broad trapped emission, which often contains multiple luminescent centers. There are various types of surface states that give rise to different energy states inside the semiconductor band gap. For $\mathrm{SnO}_{2}$, the trapped emission is complicated. For example, $\mathrm{SnO}_{2}$ nanocrystalline powder has two distinct PL emissions at 400 and $430 \mathrm{~nm}$ [2]. Three emission peaks at 439, 486 and 496 $\mathrm{nm}$ were observed from the as-synthesized $\mathrm{SnO}_{2}$ nanoribbons [19]. Two main emissions at 452 and $560 \mathrm{~nm}$ were 
found by cathodoluminescence (CL) spectroscopy for the $\mathrm{SnO}_{2}$ nanowires and nanobelts grown on $\mathrm{Al}_{2} \mathrm{O}_{3}, \mathrm{SiO}_{2}$ and $\mathrm{Si}$ substrates[20]. Up to now, the mechanisms of observed emissions are not yet clear. However, they should be associated with defect energy levels within the band gap of $\mathrm{SnO}_{2}$. Oxygen vacancies are well known to be the most common defects in oxides and usually act as radiative centre in luminescence processes. Thus, the nature of the transition is tentatively ascribed to oxygen vacancies, $\mathrm{Sn}$ vacancies or $\mathrm{Sn}$ interstitials, which form a considerable number of trapped states within the band gap[20-24]. In this work, all the $\mathrm{SnO}_{2}$ nanocrystalline powders heated at different temperatures and all samples were excited with a dominant emission peaks which was observed at $417 \mathrm{~nm}$ (blue) ascribed as luminescence centers. However, in our studies, emission maximum of $417 \mathrm{~nm}$ is lower than the band gap of the $\mathrm{SnO}_{2}$ bulk of 3.6 $\mathrm{eV}$. These peaks can be attributed to electron transition, mediated by defects levels in the band gap, such as oxygen vacancies and the luminescence centers formed by such tin interstitials or dangling in the presence of $\mathrm{SnO}_{2}$ nanocrystals. From the Fig 4, it represents rutile tetragonal $\mathrm{SnO}_{2}$ structure by a tin atom at the centre and surrounded by six oxygen atoms in the vertex. The tin atom is bonded to four oxygen $\mathrm{Sn}_{1} \mathrm{O}_{1}(4 \mathrm{x})$ atoms with the same bond length in the basal plane and another two apical oxygen formed $\mathrm{SnO}(2 \mathrm{x})$ atoms with different bond length as mentioned in the Table 1 . The Sn-O bond length shows a very high for as-prepared nanocrystalline $\mathrm{SnO}_{2}$, it was strong and maximum emission intensity is appeared at $417 \mathrm{~nm}$. When the temperatures increased, the bond length is decreased due to the reduction of oxygen vacancies. So the emission intensity is also decreased and the peak is shifted towards red shift. In addition to study the effect of the heating temperature on the photoluminescence (PL) intensity at $417 \mathrm{~nm}$ of the $\mathrm{SnO}_{2}$ nanocrystalline powders was also investigated as shown in Fig. 7.

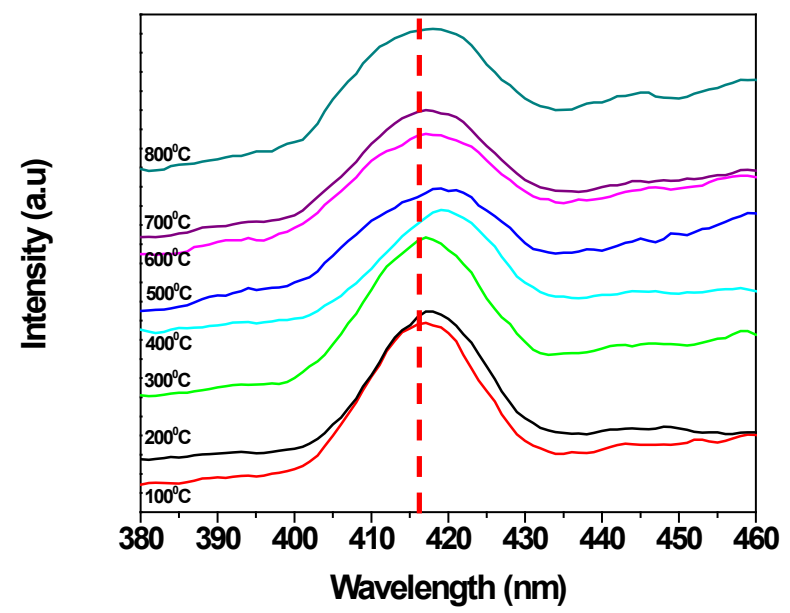

Figure 6. Photoluminescence emission spectra of $\mathrm{SnO}_{2}$ heated at different temperatures $(\lambda \mathrm{ex}=265 \mathrm{~nm})$

It can be observed that the annealing temperature is increased from 200 to $800^{\circ} \mathrm{C}$, the intensity of the $417 \mathrm{~nm}$ peak started to decrease slowly due to the crystallite size of $\mathrm{SnO}_{2}$ powders becomes larger (from 9 to $43 \mathrm{~nm}$ ). A decrease in PL intensity $(417 \mathrm{~nm})$ can occur due to the reductions of the ratio between surface area and volume and concentration of oxygen vacancies[25]. The reduction of concentration of the oxygen vacancies is due to in-diffused oxygen recombining with oxygen vacancies[26]. We conclude that based on the above discussion, the oxygen vacancies is really play an important role on PL for emission spectrum which is due to the recombination of the electrons with holes in the valence band.

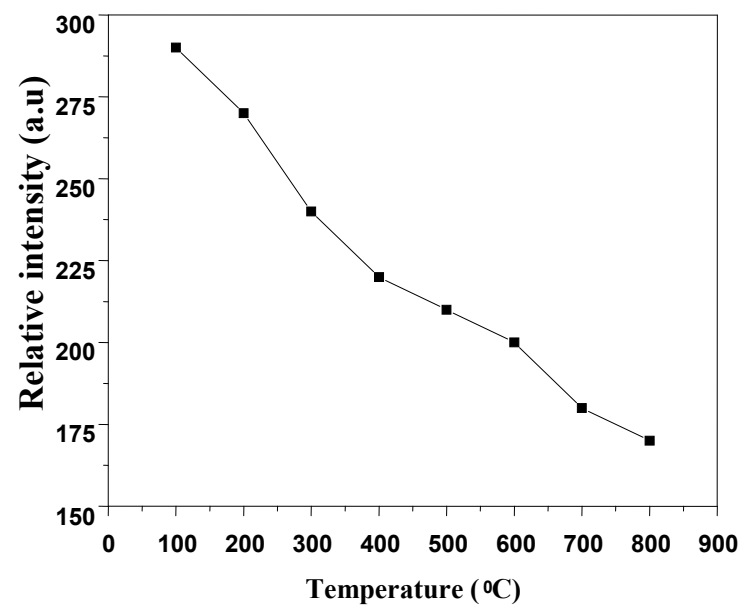

Figure 7. Temperature dependence of relative photoluminescence intensity at $417 \mathrm{~nm}$

\section{Conclusions}

In summary, tin oxide $\left(\mathrm{SnO}_{2}\right)$ nanocrystalline powders are successfully synthesized by a simple precipitation method obtained at $100^{\circ} \mathrm{C}$ by a reaction of $\mathrm{SnCl}_{2}$ and NH3. The XRD patterns confirmed that $\mathrm{SnO}_{2}$ nanocrystalline powders possess a tetragonal rutile structure. The average crystallite size of the nanopowders are in the nanometer range, upon increasing the temperatures the crystallite size of the $\mathrm{SnO}_{2}$ was found to increase from 9 to $43 \mathrm{~nm}$. The heating effect has influence the size of the crystallite on the nanocrystal powders. When samples were heated, the lattice parameters and dislocation density were decreased, so a smaller number of unit cells start to grow. Then, the obtained Rietveld refinement index value with weight percentage is $\sim 11 \%$, R Bragg value is $\sim 1 \%$ and good of fitness is $\sim 1 \%$ having good agreement in earlier reports. Photoluminescence emission exhibits a band at $417 \mathrm{~nm}$. It is related to the recombination of electrons in singly occupied oxygen vacancies with photoexcited holes in the valence band. Then the heating temperature can also affect the luminescence process, the emission resulting in the decrease in the oxygen vacancies, as revealed by the decrease in luminescence at $417 \mathrm{~nm}$.

\section{ACKNOWLEDGEMENTS}

One of the authors, L.C.Nehru would like to thank University Grants Commission (UGC), India for the financial 
support under Research Fellowship in Sciences to Meritorious Students (RFSMS) to carry out this research work at School of Physics, Alagappa University, Karaikudi-3, India.

\section{REFERENCES}

[1] A.Cabot, J. Aribiol, E.Rossinyol, J.R.Morante, Fanglin Chen and Meilin Liu, Electrochemical and solid-state letters, 2004, 7(5), G93.

[2] Feng Gu, Shu Fen Wang, Chun Feng Song, Meng Kai Lu, Yong Xin Qi, Guang Jun Zhou, Dong Xu, Duo Rong Yuan, Chemical Physics Letters, 2003, 372, 451.

[3] Feng Gu, Shu Fen Wang, Meng Kai Lu, Guang Jun Zhou, Dong Xu and Duo Rong Yuan, J. Phys. Chem. B, 2004, 108, 8119.

[4] M.M. Bagheri-Mohagheghi,_N. Shahtahmasebi, M.R. Alinejad, A. Youssefi, M. Shokooh-Saremi, Physica B, 2008, $403,2431$.

[5] Jun-Jie Zhu, Jian-Min Zhu, Xue-Hong Liao, Jiang-Lin Fang, Miao-Gao Zhou, Hong-Yuan Chen, Materials Letters, 2002, 5312.

[6] T. Krishnakumar, Nicola Pinna, K. Prasanna Kumari, K. Perumal, R. Jayaprakash, Materials Letters, 2008, 62, 3437.

[7] Zhijie Li, Wenzhong Shen, Xue Zhang, Limei Fang and Xiaotao $\mathrm{Zu}$, Colloids and surfaces A: Physicochem. Eng. Aspects, 2008, 327, 17.

[8] Z. Ying, Q. Wan, Z.T. Song, S.L. Feng, Mater. Lett. 2005, 59, 1670.

[9] Y. Liu, J. Dong, M. Liu, Adv. Mater. 2004, 16, 353.

[10] Ki Chang Song, Yong Kang, Materials Letters, 2000, 42, 283.

[11] H. Hallila, P. Méninia, and H. Auberta, Procedia Chemistry, 2009, 1, 935.
[12] J. Zhang, L. Gao, Chem. Lett, 2003, 32, 458.

[13] H. Yang, X. Song, X. Zhang, W. Ao, G. Qui, Mater. Lett, 2003, 57, 3124.

[14] S. Velumani, Xavier Mathew, P. J. Sebastian, Sa. K. Narayandass, D. Mangalaraj, Solar Energy Materials \& Solar cells, 2003, 76, 347.

[15] Preetam Sing, Ashvani Kumar, Ajay Kaushal, Davinder Kaur, Ashish Pandey and R N Goyal, Bull. Mater. Sci., 2008, 31(3), 573.

[16] J. Joseph, V. Mathew and K.E. Abraham, Crys. Res. Technol, 2006, 41(10), 1020.

[17] Evandro A. de Morais, Luis V.A. Scalvi, Alberto A. Cavalheiro, Américo Tabata, José Brás B. Oliveira, Journal of Non-Crystalline Solids, 2008, 354, 4840.

[18] A.P. Maciel, P.N. Lisboa-Filho, E.R. Leite, C.O. Paiva-Santos, W.H. Schreiner, Y. Maniette, E. Longo, Journal of the European Ceramic Society, 2003, 23, 707.

[19] Q Hu, X L Ma, N G Shang, Z Y Xie, N B Wong, C S Lee and S T Lee, J. Phys. Chem. B, 2002, 106, 3823.

[20] D Calestani, L Lazzarini, G Salviati and M Zha, Cryst.Res. Technol, 2005, 40, 937.

[21] J H He, T H Wu, C L Hsin, K M Li, L J Chen, Y L Chueh, L J Chou and Z L Wang, Small, 2006, 2, 116.

[22] J Q Hu, X L Ma, N G Shang, Z Y Xie, N B Wong, C S Lee and S T Lee, J. Phys. Chem. B, 2002, 106, 3823.

[23] H W Kim, N H Kim, J H Myung and S H Shim, Phys. Status Solidi A, 2005, 2021758.

[24] S Brovelli, N Chiodini, F Meinardi, A Lauria and A Paleari, Appl. Phys. Lett, 2006, 89, 153126.

[25] Feng Gu, Shu Fen Wang, Chun Feng Song, Meng Kai Lü, Ypog Xin Qi, Guang Jun Zhou, Dong Xu and Duo Rong Yuan, Chemical Physics Letters, 2003, 372, 451.

[26] K. Vanheusden, W.L. Warren, C.H. Seager, D.R. Tallant, J.A. Voigt and B.E.Gnade, J. Appl. Phys. 1996, 79, 7983. 